\title{
VARIABILIDAD DIURNA DE LA PRESIÓN INTRAOCULAR
}

\section{DIURNAL VARIABILITY OF INTRAOCULAR PRESSURE}

\author{
JAÉN-DÍAZ JI ${ }^{1}$, CORDERO-GARCÍA B ${ }^{1}$, LÓPEZ-DE-CASTRO ${ }^{1}$, DE-CASTRO-MESA C ${ }^{1}$, \\ CASTILLA-LÓPEZ-MADRIDEJOS $\mathrm{F}^{1}$, BERCIANO-MARTÍNEZ $\mathrm{F}^{1}$
}

\section{RESUMEN}

Objetivo: Comprobar y cuantificar la posible variación de los valores de presión intraocular (PIO) en sujetos sanos, entre la mañana y la tarde.

Métodos: Estudio descriptivo y transversal realizado en un Centro de Salud entre los consultantes habituales de una consulta de medicina general. Se realizaron dos tomas de presión intraocular el mismo día (la primera entre las 8 y las 9 horas y la segunda entre las 17 y las 18 horas) utilizando un tonómetro de aplanación tipo Perkins. Los sujetos (119 mujeres y 101 hombres, edad media de 46,3 años con un rango entre 14 y 83) estaban distribuidos proporcionalmente en cuatro grupos de edad (14-24, 25-44, 45-64 y >65 años).

Resultados: Los valores medios de PIO fueron: Ojo derecho en la mañana y en la tarde 13,29 y 11,81 ( $\mathrm{p}<0,001)$ respectivamente y Ojo izquierdo en la mañana y en la tarde 13,48 y $12,04(\mathrm{p}<0,001)$. La PIO fue significativamente mayor en hombres que en mujeres, teniendo una correlación muy débil con la edad.

Conclusiones: Constatamos una diferencia en los valores de PIO entre la mañana y la tarde, de aproximadamente $1,5 \mathrm{mmHg}$. El momento del día en que se determina la PIO debería contemplarse como una variable más en los estudios de hipertensión ocular y glaucoma que la determinan.

Palabras clave: Presión intraocular, glaucoma, variabilidad, tonometría, Atención Primaria.

\section{ABSTRACT}

Objective: To assess whether there are variations in the intraocular pressure (IOP) between the morning and afternoon in healthy subjects.

Methods: This study was carried out in a Primary Care Health Center among usual patients of general practitioners. Two measurements of the intraocular pressure were assessed on the same day (between 8 and 9 a.m. and between 5 and 6 p.m.) using a Perkins applanation tonometer. The subjects (119 females and 101 males) had a mean age of 46.3 years, with ages ranging between 14 and 83 years. They were proportionally distributed among 4 age groups (14-24, 25-44, 45-64 and $>65$ years).

Results: The IOP mean values were: right eye, morning time and afternoon, 13.29 and $11.81 \mathrm{~mm}$ $\mathrm{Hg}$, respectively $(\mathrm{p}<0.001)$; left eye, morning time and afternoon, 13.48 and $12.04 \mathrm{~mm} \mathrm{Hg}(\mathrm{p}<0.001)$. The IOP was significantly higher in males than in females, and showed a weak association with age. Conclusions: We found a diurnal variation of IOP of approximately $1.5 \mathrm{~mm} \mathrm{Hg}$. The time of day when the IOP is measured needs to be considered as a variable in research in ocular hypertension and glaucoma (Arch Soc Esp Oftalmol 2007; 82: 675680).

Key words: Intraocular pressure, glaucoma, variability, tonometry, Primary Care.

\footnotetext{
Recibido: 26/6/06. Aceptado: 20/9/07.

Centro de Salud Docente Santa M. ${ }^{a}$ de Benquerencia. Toledo. España.

1 Licenciado en Medicina. Especialista en Medicina Familiar y Comunitaria.

Correspondencia:

José Ignacio Jaén Díaz

Centro de Salud Santa María de Benquerencia

C/. Guadarrama, s/n

45007 Toledo

España

E-mail: ijd02@hotmail.com
} 


\section{INTRODUCCIÓN}

La presión intraocular (PIO) puede considerarse como la resultante del equilibrio entre la producción y la eliminación del humor acuoso (1). Por mecanismos todavía no bien conocidos, su aumento se considera uno de los principales factores de riesgo para desarrollar glaucoma (2). Aunque se acepta un valor arbitrario máximo de normalidad de $21 \mathrm{mmHg}$ (3), hay que tener en cuenta que, como otros muchos parámetros biológicos, se trata de un valor variable que se encuentra influenciado por muchos factores: edad (4), sexo (5), raza (6), consumo de tabaco (7), problemas oculares locales (8), obesidad (9), cambios hormonales (10), ejercicio físico (11), etc.

Además, parece seguir un ritmo circadiano, aún no claramente establecido en los humanos, con un pico máximo durante la mañana y otro mínimo al final de la tarde; sin tener en cuenta las todavía menos claras oscilaciones que acontecen durante la noche (12-15).

El conocimiento de estos cambios cíclicos de la PIO, así como de la magnitud de los mismos y de los posibles factores que pueden modificarlos, tiene un importante valor no solo desde el punto de vista de la investigación sino también desde el punto de vista clínico en cuanto al diagnóstico y al manejo de los pacientes hipertensos oculares y con glaucoma. Como ejemplo, en un estudio de prevalencia de hipertensión ocular y glaucoma realizado por nuestro grupo hace cinco años y desarrollado en horario de tarde, llamaba la atención la existencia de valores medios de PIO sensiblemente inferiores a los descritos en trabajos similares (16).

El objetivo del presente trabajo fue comprobar si existían diferencias en los valores de presión ocular de una población a las horas del día en que se realizaba la medida y cuantificar dicha diferencia.

\section{SUJETOS, MATERIAL Y MÉTODOS}

Se trata de un estudio descriptivo y transversal, realizado en una zona de salud urbana de la ciudad de Toledo. Se incluyeron personas residentes en la zona, distribuidas, proporcionalmente, en cuatro grupos de edad (14-24 años, 25-44, 45-64 y $\geq 65$ ), no diagnosticadas previamente de glaucoma o hipertensión ocular y pertenecientes a la población consultante del Centro de Salud. Los pacientes, elegidos entre los citados en la consulta por cualquier motivo, entre las 8 y las 9 horas de la mañana, eran informados del estudio y una vez obtenido su consentimiento, eran sometidos a una determinación de su PIO y citados para una nueva determinación, ese mismo día, entre las 17 y las 18 horas.

La medición de la PIO se realizó mediante dos tomas seriadas de la misma, en ambos ojos (primero en el derecho y después en el izquierdo), tras la instilación de una gota de anestésico y fluoresceína (solución comercial de fluoresceína sódica al 0,25\% y oxibuprocaína clorhidrato al $0,4 \%$ ), con el paciente en posición sentada y erecta, utilizando un tonómetro de aplanación tipo Perkins (modelo MK2, Clement Clarke Ltd., England) y eligiendo, para cada ojo, el valor mas alto de las dos tomas. Todas las medidas fueron hechas por un único explorador con amplia experiencia previa en la técnica.

Los datos obtenidos fueron introducidos en una base del programa SPSS 9.0 para Windows (SPSS por Windows, SPSS Inc, Chicago, USA), que se empleó para su tratamiento estadístico. Para la descripción de los resultados se han utilizado los parámetros habituales (media, desviación estándar, porcentaje) y los intervalos de confianza del $95 \%$. En el análisis estadístico, una vez comprobada la nonormalidad de la PIO mediante la prueba de Kolmogorov-Smirnov, se han utilizado los tests de Mann-Whitney (U), Kruskall-·Wallis $\left(\chi^{2}\right)$ y Wilcoxon (Z), este último para comparar datos apareados. También se calcularon los coeficientes de correlación rho $(\rho)$ de Spearman.

\section{RESULTADOS}

La muestra final la componen 220 personas, 119 mujeres $(54,1 \%)$ y 101 hombres $(45,9 \%)$. La edad media es de 46,3 años, con un rango entre 14 y 83. La edad de los hombres fue mayor que la de las mujeres (49,3 versus 43,7; $\mathrm{p}<0,05)$.

Los valores de PIO obtenidos en cada ojo, en la mañana y en la tarde, se muestran en la figura 1. Resultan ligeramente mayores los valores de PIO en el ojo izquierdo que en el derecho y en la mañana que en la tarde $(\mathrm{p}<0,001)$. La diferencia entre la PIO de mañana y tarde fue de $+1,48 \mathrm{mmHg}\left(\mathrm{IC}_{95 \%}\right.$ $1,24-1,72)$ en ojo derecho y de $+1,44 \mathrm{mmHg}\left(\mathrm{IC}_{95 \%}\right.$ 1,21-1,67) en el izquierdo.

La correlación entre la edad y la PIO es muy débil (rho $=+0,15$ y $+0,13$ para ojo derecho e izquierdo respectivamente). En la tabla I se muestra 


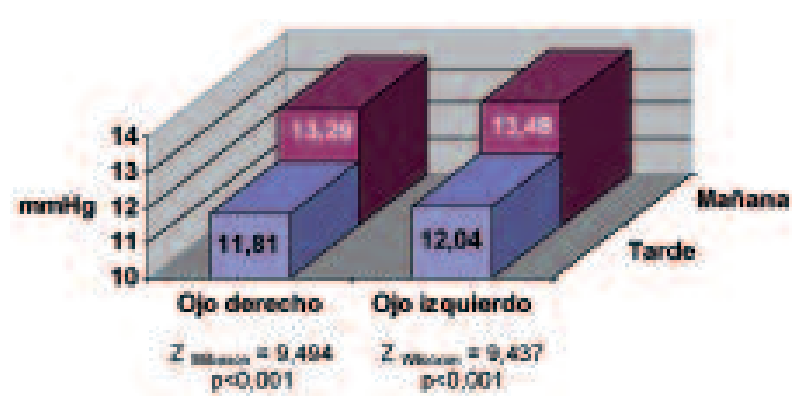

Fig. 1: Valores de presión intraocular según horario de la toma.

la PIO según los tramos de edad establecidos, observándose en general ligeros aumentos de PIO al aumentar el tramo etario, pero con diferencias no significativas.

Como puede verse en la figura 2, la PIO resultó mayor en hombres que en mujeres. Esta observación se constata en tres de los cuatro grupos etarios.

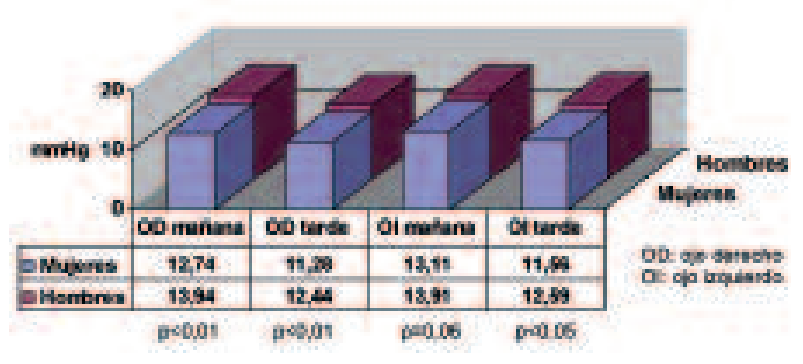

Fig. 2: Valores de PIO según sexo.

\section{DISCUSIÓN}

Nuestro trabajo confirma que existen diferencias entre las cifras de PIO detectadas a primera hora de la mañana y a primera hora de la tarde, en el mismo grupo de población y en determinaciones realizadas el mismo día.

Las PIOs medias halladas son similares a las encontradas en un estudio de prevalencia previo que se realizó en la misma zona de salud (16) con una metodología similar, pero en un grupo de edad de mayores de 40 años. En aquel no se encontraron diferencias de PIO en función de la edad ni del sexo, mientras que en el actual sí existen, en función de éste último aspecto. La influencia de la edad y el sexo en los valores de PIO es, habitualmente, positiva aunque no todos los estudios que la evalúan lo constatan. Así, y centrándonos en la población española, existen dos trabajos recientes que la encuentran en un caso (17) y en el otro no (18). Es posible que la distinta metodología empleada y la diferente población de estudio seleccionada sean los responsables de estas discrepancias.

La diferencia de PIO entre ambos ojos ya ha sido descrita previamente $(19,20)$ y puede deberse a que siempre se toma la PIO primero en un ojo y luego en otro; siendo conocido el hecho de que la movilización o el masaje de un ojo puede provocar cambios en la PIO del otro (21). En cualquier caso, la diferencia tiene escasa relevancia clínica, ya que está en torno a $0,2 \mathrm{mmHg}$.

Aunque no completamente aclarado, es bien conocido el hecho de que los valores de PIO, al

Tabla I. Valores de presión intraocular (PIO) según tramos de edad, ojo y orario de toma

\begin{tabular}{lccccc}
\hline Ojo y hora & Grupo etario & $\mathrm{N}$ & Media PIO & Desviación típica & Significación Estadística* \\
\hline Derecho & $14-24$ & 41 & 12,54 & 2,684 & 5,355 \\
Mañana & $25-44$ & 59 & 13,17 & 2,919 & $\mathrm{p}=0,148$ \\
& $45-64$ & 63 & 13,25 & 2,967 & \\
Izquierdo & $>65$ & 57 & 14,00 & 3,454 & 4,894 \\
Mañana & $14-24$ & 41 & 12,63 & 2,817 & $\mathrm{p}=0,180$ \\
& $25-44$ & 59 & 13,20 & 2,815 & 0,860 \\
Derecho & $45-64$ & 63 & 13,90 & 3,031 & $\mathrm{p}=0,835$ \\
Tarde & $>65$ & 57 & 13,89 & 3,534 & 1,154 \\
& $14-24$ & 41 & 11,54 & 2,550 & $\mathrm{p}=0,764$ \\
Izquierdo & $25-44$ & 59 & 11,76 & 2,445 & 2,923 \\
Tarde & $45-64$ & 63 & 11,81 & 3,061 & 2,675 \\
\end{tabular}

* Test de Kruskall-Wallis. 
igual que los de otros tantos parámetros y variables biológicas, siguen un ritmo circadiano, con oscilaciones en su valores a lo largo de las 24 horas del día. De manera general aunque no constante, en sujetos sanos, los valores mas altos de PIO se obtienen al principio del día, al levantarse, descendiendo paulatinamente durante el día hasta la hora de dormir y volviéndose a elevar de manera también progresiva durante la noche. Las diferencias entre los valores máximos y mínimos pueden llegar a los 8 $\mathrm{mmHg}$ (22). Este ciclo podría estar alterado o ser diferente en sujetos con glaucoma (20).

El estudio de la variación diurna de la PIO, que sepamos, ha sido escasamente tratado en la población española, habiendo encontrado solamente un trabajo publicado al respecto (23) en la población atendida en un servicio de Oftalmología.

Nuestro trabajo demuestra, en nuestra población (población general atendida en una consulta de un centro de salud), que la PIO es menor (aproximadamente $1,5 \mathrm{mmHg}$ ) a primera hora de la tarde que de la mañana y tanto en hombres como en mujeres. Saccá y cols (14) encuentran, en sujetos no glaucomatosos, diferencias similares entre las determinaciones realizadas a las $8 \mathrm{~h}$ de la mañana y a las $16 \mathrm{~h}$ de la tarde.

La primera consecuencia derivada de éste hecho, y en ello coincidimos con otros autores (12), es que aquellos pacientes que presenten PIOs límites a primeras horas de la tarde deberían ser reevaluados en horario matutino. La constatación de esta variación obliga, también, a ser cautos a la hora de interpretar los valores de la presión intraocular detectados tanto en población sana como glaucomatosa y tanto desde el punto de vista diagnóstico como de control del tratamiento y a relacionarlos con la hora del día en la que se realiza, y refrendan el hecho conocido de que las tomas aisladas de PIO aportan poca información sobre los valores máximos, el rango de variación y la variabilidad de la misma (20). De hecho creemos que este aspecto (la hora de determinación de la PIO) debería contemplarse como una variable más en los estudios epidemiológicos relacionados con la hipertensión ocular y el glaucoma.

\section{BIBLIOGRAFÍA}

1. Vaughan D, Asbury T. Oftalmología general. México: El Manual Moderno; 1976; 212.

2. Fernández PC. Glaucoma. Medicine 1998; 7: 4770-4777.
3. Jackson C, Loane M, Glasson W. Assessing for glaucoma in general practice. Aust Fam Physician 1996; 25: $1405-$ 1411.

4. Wensor MD, McCarty CA, Stanislavsky YL, Livingston PM, Taylor HR. The prevalence of glaucoma in the Melbourne Visual Impairment Project. Ophthalmology 1998; 105: 733-739.

5. Qureshi IA. Intraocular pressure: a comparative analysis in two sexes. Clin Physiol 1997; 17: 247-255.

6. Tielsch JM, Sommer A, Katz J, Ragal R, Quiggley HA, Javitt J. Racial variations in the prevalence of primary open-angle glaucoma. The Baltimore Eye Survey. JAMA 1991; 266: 369-374.

7. Morgan RW, Drance SM. Chronic open-angle glaucoma and ocular hypertension. An epidemiological study. Br J Ophthalmol 1975; 59: 211-215.

8. Shah S. Accurate intraocular pressure measurement-the myth of modern ophthalmology? Ophthalmology 2000; 107: 1805-1806.

9. Dos Santos MG, Makk S, Berghold A, Eckhardt M, Haas A. Intraocular pressure difference in Goldmann applanation tonometry versus Perkins hand-held applanation tonometry in overweight patients. Ophthalmology 1998; 105: 2260-2263.

10. Sator MO, Gruber DM, Joura EA. Hormonal influences on intraocular pressure. Lancet 1996; 348: 761-762.

11. Passo MS, Goldberg L, Elliot DL, Van Buskirk EM. Exercise conditioning and intraocular pressure. Am J Ophthalmol 1987; 103: 754-757.

12. Pointer JS. The diurnal variation of intraocular pressure in non-glaucomatous subjects: relevance in a clinical context. Ophthalmic Physiol Opt 1997; 17: 456-465.

13. Liu JH. Circadian rhythm of intraocular pressure. Glaucoma 1998; 7: 141-147.

14. Sacca SC, Rolando M, Marletta A, Macri A, Cerqueti P, Ciurlo G. Fluctuations of intraocular pressure during the day in open-angle glaucoma, normal-tension glaucoma and normal subjects. Ophthalmologica 1998; 212: 115 119.

15. Liu JH, Kripke DF, Hoffman RE, Twa MD, Loving RT, Rex KM et al. Nocturnal elevation of intraocular pressure in young adults. Invest Ophtalmol Vis Sci 1998; 39: 27072712.

16. Jaén Díaz J, Sanz Alcolea I, López de Castro F, Pérez Martínez T, Ortega Campos P, Corral Morales R. Glaucoma e hipertensión ocular en atención primaria. Aten Primaria 2001; 28: 23-30.

17. Zafra Pérez JJ, Villegas Pérez MP, Canteras Jordana M, Miralles de Imperial J. Presión intraocular y prevalencia de glaucoma oculto en una población de la región de Murcia. Arch Soc Esp Oftalmol 2000; 75: 171-178.

18. Puell Marín MC, Romero Martín M, Domínguez Carmona M. Presión intraocular en adultos jóvenes: su relación con el consumo de tabaco y cafeína. Arch Soc Esp Oftalmol 1998; 73: 255-262.

19. Noel C, Kalco AM, Romanet JP, Montmayeur A, Buguet A. Twenty-four-hour time course of intraocular pressure in healthy and glaucomatous Africans: relation to sleep patterns. Ophthalmology 2001; 108: 139-144.

20. Wilensky JT, Gieser DK, Dietsche ML, Mori MT, Zeimer $R$. Individual variability in the diurnal intraocular pressure curve. Ophthalmology 1993; 100: 940-944. 
21. Cooper RL, Beale DG, Constable IJ, Grose GC. Continual monitoring of intraocular pressure, respiration, and eye movements on continual recording of intraocular pressure in the rabbit, dog and man. Br J Ophthalmol 1979; 63: 799-804.

22 David R, Zangwill L, Briscoe D, Dagan M, Yaget R, Yas- sur Y. Diurnal intraocular pressure variations: an analysis of 690 diurnal curves. Ophthalmol 1992; 76: 280-283.

23. García García AC, Jordano Pérez J, Rodríguez Escobar JJ, Sánchez Rejón MA. Influencia del ritmo circadiano en la presión intraocular. Arch Soc Esp Oftalmol 1979; 39: 798-803. 